\title{
Effects of percutaneous angioplasty on kidney function and blood pressure in patients with atherosclerotic renal artery stenosis
}

\author{
Suhyun Kim ${ }^{1, *(1)}$, Mi Jeoung Kim ${ }^{1,2, *(1)}$, Jeunseok Jeon ${ }^{1(\mathbb{D})}$, Hye Ryoun Jang ${ }^{1(\mathbb{D}}$, Kwang Bo Park ${ }^{3}$, \\ Wooseong Huh ${ }^{1 \text { (DD }}$, Young Soo $\mathrm{Do}^{3}$, Yoon-Goo Kim${ }^{1}$, Dae Joong Kim ${ }^{1}$, Ha Young $\mathrm{Oh}^{1}$, Jung Eun Lee ${ }^{1(\mathbb{D})}$ \\ ${ }^{1}$ Division of Nephrology, Department of Medicine, Samsung Medical Center, Sungkyunkwan University School of Medicine, Seoul, Korea \\ Department of Internal Medicine, Incheon Sarang Hospital, Incheon, Korea \\ ${ }^{3}$ Department of Radiology, Samsung Medical Center, Sungkyunkwan University School of Medicine, Seoul, Korea
}

\begin{abstract}
Background: Previous randomized controlled trials of revascularization for atherosclerotic renal artery stenosis (ARAS) were not successful. We investigated the effects of percutaneous transluminal angioplasty with stent insertion (PTA/S) on kidney function and blood pressure (BP) control in patients with ARAS.

Methods: From 2000 to 2017, 47 subjects who underwent PTA/S for ARAS were identified. A high-risk group was defined, composed of patients having one or more of the following clinical presentations: pulmonary edema, refractory hypertension, and rapid deterioration of kidney function. Subjects who met the criteria of 'kidney function improvement' or 'hypertension improvement' after PTA/S were classified as responders.

Results: Twenty-one (44.7\%) subjects were classified into the high-risk group. Two subjects $(8.0 \%)$ in the low-risk group $(n=25)$ and 5 subjects $(27.8 \%)$ in the high-risk group $(n=18)$ showed improvement in kidney function after PTA/S $(P=0.110)$. In patients with rapid decline of kidney function, estimated glomerular filtration rate improved from 28 (interquartile range [IQR], 10-45) mL/min/1.73 $\mathrm{m}^{2}$ to 41 (IQR, 16-67) $\mathrm{mL} / \mathrm{min} / 1.73 \mathrm{~m}^{2}$ at 4 months after PTA/S, although the difference was not significant $(P=0.084)$. Regarding BP control, 9 (36.0\%) and $14(77.8 \%)$ subjects showed improvement after PTA/S in the low- $(n=25)$ and high-risk $(n=18)$ groups, respectively $(P=0.007)$. In patients with refractory hypertension, the systolic BP dropped from 157 (IQR, 150-164) mmHg to 140 (IQR, 131148) $\mathrm{mmHg}$ at 4 months after PTA/S $(P=0.005)$. Twenty-five subjects were defined as responders and comprised a significant proportion of the high-risk group $(P=0.004)$.

Conclusion: PTA/S might improve BP control and kidney function in patients with ARAS presenting with highrisk clinical features. The optimal application of PTA/S should be based on individual assessment of the clinical significance of renal artery stenosis.
\end{abstract}

Keywords: Angioplasty, Blood pressure, Glomerular filtration rate, Renal artery stenosis

Received November 30, 2018; Revised March 14, 2019; Accepted March 28, 2019

Edited by Dae Ryong Cha, Korea University, Ansan, Korea

Correspondence: Jung Eun Lee

Division of Nephrology, Department of Medicine, Samsung Medical Center, Sungkyunkwan University School of Medicine, 81 Irwon-ro, Gangnam-gu, Seoul 06351, Korea. E-mail: jungeun34.lee@samsung.com

*Suhyun Kim and Mi Jeoung Kim contributed equally to this work.

Copyright (C) 2019 by The Korean Society of Nephrology

(a) This is an open-access article distributed under the terms of the Creative Commons Attribution Non-Commercial License (http://creativecommons.org/ licenses/by-nc-nd/4.0/), which permits unrestricted non-commercial use, distribution, and reproduction in any medium, provided the original work is properly cited. 


\section{Introduction}

Atherosclerotic renal artery stenosis (ARAS) is the most common cause of renal artery stenosis (RAS) and is associated with severe comorbidities such as resistant hypertension, ischemic nephropathy, and end-stage renal disease $[1,2]$. Common therapeutic strategies for RAS include medical therapy, angioplasty with or without stenting, and bypass surgery. Percutaneous transluminal angioplasty (PTA) is a highly effective modality for correction of RAS. However, two recent randomized controlled trials (RCT) of PTA for ARAS failed to demonstrate any advantages compared with conventional medical therapy regarding renal protection or prevention of cardiovascular events [3-6]. Nevertheless, ARAS frequently progresses despite adequate medical management and results in irreversible renal atrophy. Thus, timely corrections of RAS through PTA with stent insertion (PTA/S) may have a beneficial effect on long-term renal outcomes in selected patients.

Current guidelines for ARAS recommend PTA as a reasonable option for patients with haemodynamically significant RAS with clinical manifestations such as resistant hypertension, unexplained pulmonary edema, or progressive chronic kidney disease $[7,8]$. However, patient selection for renal revascularization is sometimes challenging, because limited comparative data are available on the specific phenotype of patients who would benefit from the procedure [9-11]. In this study, we investigated the effects of renal revascularization in terms of kidney function and blood pressure (BP) control in patients who received PTA/S for ARAS, classifying subjects into lowrisk and high-risk groups. High-risk was defined by clinical presentation including a history of pulmonary edema, refractory hypertension, or rapid deterioration of kidney function accompanied by RAS.

\section{Methods}

\section{Study population}

We retrospectively identified 62 subjects with RAS who had undergone PTA/S at a 2,000-bed tertiary hospital between January 2000 and October 2017. We excluded subjects with RAS related to kidney transplantation, fibromuscular dysplasia, or Takayasu's disease; subjects who had a history of previous PTA/S; or those who were lost to follow-up within two weeks after the procedures. Ultimately, 47 subjects were included in this study. The study protocol was reviewed and approved by the Institutional Review Board (IRB) of Samsung Medical Center (2017-11-164). The obtain written informed consent from the participants for this retrospective study was waived by IRB.

\section{Data and definitions}

The following patient data were collected at the time of PTA/S: age, sex, smoking status, body mass index (BMI), comorbidities (diabetes mellitus, coronary artery disease, peripheral artery disease, stroke), systolic BP (SBP), diastolic BP (DBP), serum albumin, total cholesterol, serum creatinine, spot urine protein-to-creatinine ratio (uPCR), targeted kidney size, contralateral kidney size, stenosis severity of the targeted kidney artery, and medication history including antihypertensive medication. Data on kidney size and stenosis severity of the renal artery were collected through a review of sonographic and angiographic records, respectively. Follow-up data for serum creatinine, SBP, DBP, and BP medical score (BPMS) were collected at one month ( \pm 2 weeks) and at 4 months ( \pm 1 month) after PTA/S.

The estimated glomerular filtration rate (eGFR) was calculated using the Chronic Kidney Disease Epidemiology Collaboration 2009 creatinine equation [12]. PostPTA acute kidney injury is defined as impairment of renal function, measured as either a $25 \%$ increase in serum creatinine from baseline or a $0.5 \mathrm{mg} / \mathrm{dL}$ increase in absolute serum creatinine value, within 48 to 72 hours of the intervention. We utilized the BPMS scoring system to estimate the antihypertensive agent potency, defined as "BPMS [13] —sum of the score of each antihypertensive agent," and by setting the equivalent dose for each ingredient that could yield one point [14-19]. Alpha-adrenergic blocking agents considered as antihypertensive agents were terazosin and doxazosin [20]. Use of diuretics was assigned as a 1 point to BRMS regardless of the ingredient and dose, assuming that the administered dose was appropriate to maintain euvolemic status.

Through a review of the medical records, we classified the study subjects into two groups: low-risk and high-risk group. The high-risk group was defined as patients with a 
history of pulmonary edema, refractory hypertension, or rapid deterioration of kidney function accompanied by RAS at baseline (the time of procedure). Pulmonary edema was defined if the chest radiographic imaging showed evidence of pulmonary edema requiring treatment with intravenous medications such as furosemide. Rapid decline of kidney function was defined if the serum creatinine level increased over $25 \%$ during the six months prior to PTA/S. Refractory hypertension was defined if the SBP was $>140 \mathrm{mmHg}$ and/or the DBP was $>90 \mathrm{mmHg}$ despite the use of three or more different classes of antihypertensive agents, including diuretics, in accordance with the European Society of Hypertension/European Society of Cardiology guidelines [21], or if the SBP was > $155 \mathrm{mmHg}$ despite the use of two or more different classes of antihypertensive agents in accordance with the Cardiovascular

Table 1. Baseline characteristics of the study subjects $(n=47)$

\begin{tabular}{|c|c|c|c|}
\hline Variable & Low-risk $(n=26)$ & High-risk (n = 21) & $P$ value \\
\hline Age (yr) & $68(58-76)$ & $66(61-75)$ & 0.983 \\
\hline Sex, male & $20(76.9)$ & $15(71.4)$ & 0.668 \\
\hline Smoking & $9(34.6)$ & $11(52.4)$ & 0.221 \\
\hline Body mass index $\left(\mathrm{kg} / \mathrm{m}^{2}\right)$ & $23.4(21.8-25.6)$ & $24.0(22.0-25.3)$ & 0.591 \\
\hline SBP (mmHg) & $134(122-141)$ & $152(143-160)$ & 0.188 \\
\hline $\mathrm{DBP}(\mathrm{mmHg})$ & $76(68-80)$ & $77(69-88)$ & $<0.001$ \\
\hline \multicolumn{4}{|l|}{ Comorbidity } \\
\hline Diabetes mellitus & $11(42.3)$ & $13(61.9)$ & 0.181 \\
\hline CAD & $16(61.5)$ & 9 (42.9) & 0.202 \\
\hline PAD & $3(11.5)$ & $7(33.3)$ & 0.086 \\
\hline Stroke & $7(26.9)$ & $4(19.0)$ & 0.731 \\
\hline Baseline eGFR (mL/min/1.73 $\left.\mathrm{m}^{2}\right)$ & $49(34-64)$ & $33(20-47)$ & 0.046 \\
\hline$<30 \mathrm{~mL} / \mathrm{min} / 1.73 \mathrm{~m}^{2}$ & $3(11.5)$ & $10(47.6)$ & 0.006 \\
\hline$\triangle \mathrm{eGFR}^{\mathrm{a}}$ & $-1.3(-4.4$ to -2.7$)$ & $-4.9(-17.1$ to -1.6$)$ & 0.011 \\
\hline Serum albumin (g/dL) & $4.1(3.8-4.4)$ & $3.8(3.6-4.2)$ & 0.118 \\
\hline Total cholesterol (mg/dL) & $149(127-168)$ & 149 (132-175) & 0.352 \\
\hline uPCR (mg/mgCr) & $0.21(0.09-0.52)$ & $0.31(0.00-0.93)$ & 0.163 \\
\hline Targeted kidney size (cm) & $10.0(9.0-10.2)$ & $9.8(9.3-10.2)$ & 0.703 \\
\hline Contralateral kidney size (cm) & $10.0(9.1-10.0)$ & $9.6(8.5-10.0)$ & 0.411 \\
\hline Targeted arterial stenosis & & & 0.108 \\
\hline$>95 \%$ & $4(15.4)$ & $1(4.8)$ & \\
\hline $75-95 \%$ & $18(69.2)$ & $19(90.5)$ & \\
\hline$<75 \%$ & $4(15.4)$ & $0(0)$ & \\
\hline Bilateral stent insertion & $2(7.7)$ & $6(28.6)$ & 0.115 \\
\hline \multicolumn{4}{|l|}{ Concomitant medication } \\
\hline ACEi/ARB & $8(30.8)$ & 9 (42.9) & 0.391 \\
\hline Diuretics & 7 (26.9) & $15(71.4)$ & 0.002 \\
\hline CCB & $18(69.2)$ & $14(66.7)$ & 0.851 \\
\hline Beta blocker & $13(50.0)$ & $18(85.7)$ & 0.010 \\
\hline Alpha blocker & $2(7.7)$ & $1(4.8)$ & 1.000 \\
\hline Other anti-hypertensive drug & $2(7.7)$ & $1(4.8)$ & 1.000 \\
\hline BPMS & $1.5(1.0-3.0)$ & $2.5(1.8-4.5)$ & 0.020 \\
\hline
\end{tabular}

Data are presented as median (interquartile range) or number (\%).

ACEi, angiotensin-converting enzyme inhibitor; ARB, angiotensin receptor blocker; BPMS, blood pressure medical score; CAD, coronary artery disease; CCB, calcium channel blocker; $\mathrm{Cr}$, creatinine; DBP, diastolic blood pressure; eGFR, estimated glomerular filtration rate; PAD, peripheral artery disease; SBP, systolic blood pressure; UPCR, urine protein-to-creatinine ratio.

${ }^{a}$ Median change in kidney function between baseline eGFR and pre-procedural eGFR. The pre-procedural eGFR is the result of the last examination within six months prior to the percutaneous transluminal angioplasty with stent insertion. 
Outcomes in Renal Atherosclerotic Lesions (CORAL) trial [4]. Patients not satisfying any of the three high-risk clinical features described above were classified into the lowrisk group.

The primary outcome was improvement of either kidney function or hypertension after PTA/S. Kidney function improvement was defined as recovery of eGFR over $20 \%$ compared to baseline at 4 months ( \pm 1 month) after PTA/S. Hypertension improvement was defined as resolution of refractory hypertension, a decrease in BPMS by more than 2 points, or interrupted use of the BP-lowering agent after 4 months ( \pm 1 month) of PTA/S. Subjects who achieved either kidney function improvement or hypertension improvement were classified as responders. All remaining subjects were classified as non-responders.

\section{Statistical analyses}

Data are presented as median and interquartile range (IQR). Baseline continuous variables were compared using the independent two sample $t$ test or the MannWhitney rank sum test according to data distribution, while categorical variables were compared using the chisquare test or Fisher's exact test. Differences in serial eGFR, SBP, DBP, and BPMS between the two groups were assessed by a linear mixed model using a specific subgroup and time as an independent variable. All statistical analyses were performed using IBM SPSS ver. 25.0 for Windows (IBM Corp., Armonk, NY, USA). A $P$ value $<0.05$ was considered statistically significant.

\section{Results}

\section{Baseline demographics and clinical characteristics of the study subjects}

The baseline characteristics of the study population ( $\mathrm{n}$ $=47$ ) are summarized in Table 1. Twenty-one subjects (44.7\%) satisfied the criteria of the high-risk group. Among these, 2 subjects presented with pulmonary edema, 7 subjects met the criteria for rapid decline of kidney function, 17 subjects met the criteria for refractory hypertension, and 5 subjects met the criteria for both rapid decline of kidney function and refractory hypertension.

The median age was $68(58-76)$ years in the low-risk group and $65(61-75)$ years in the high-risk group. The baseline eGFR value was $49(34-64) \mathrm{mL} / \mathrm{min} / 1.73 \mathrm{~m}^{2}$ in the low-risk group and $33(20-47) \mathrm{mL} / \mathrm{min} / 1.73 \mathrm{~m}^{2}$ in the high-risk group. The median changes in kidney function during the six months prior to PTA/S were $-1.3(-4.4$ to $2.7)$ and $-4.9(-17.1$ to -1.6$)$ in the low-risk and high-risk groups, respectively $(P=0.011)$. As expected, the highrisk group had higher BP $(P<0.001)$ and a larger proportion of subjects taking three or more anti-hypertensive
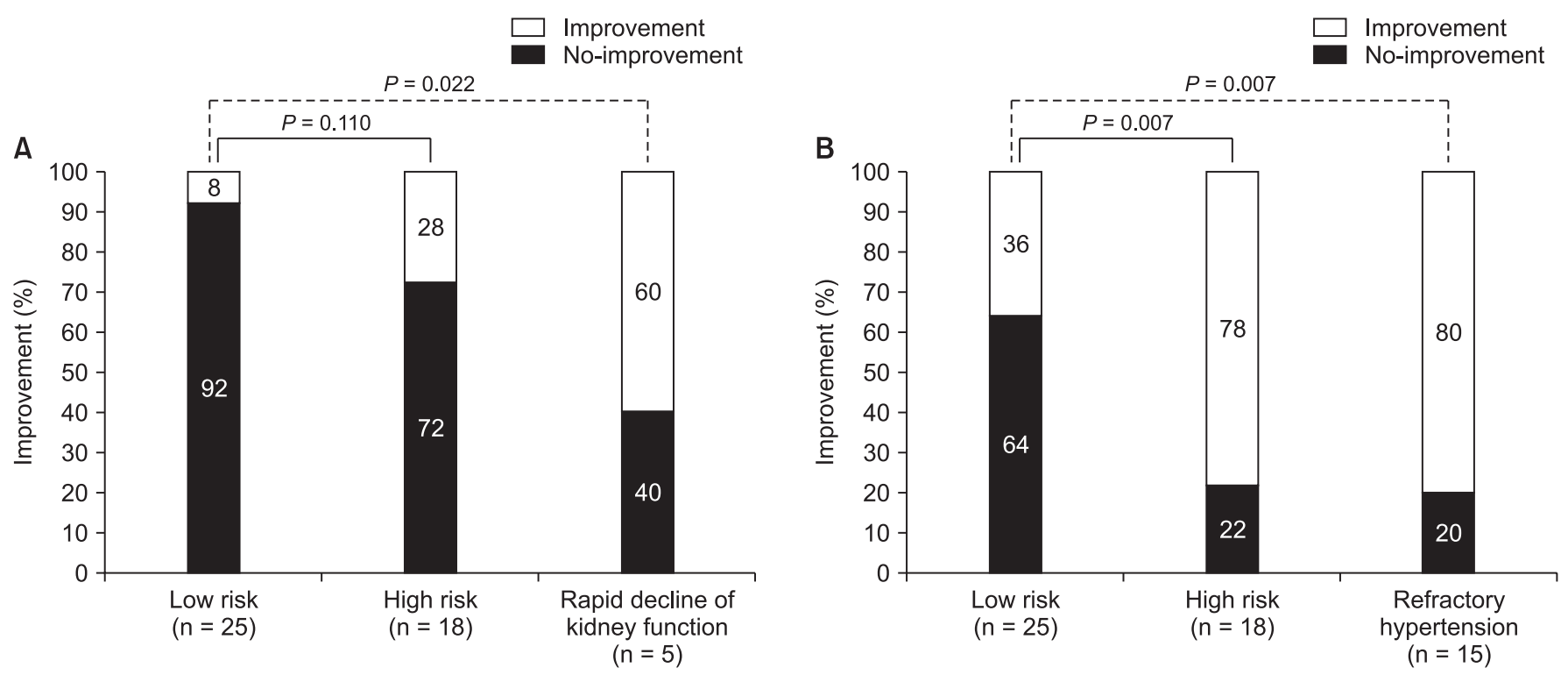

Figure 1. Proportion of subjects who showed kidney function improvement (A) or improvement of hypertension (B) at 4 months ( \pm 1 month) after percutaneous transluminal angioplasty with stent insertion. 
agents $(P=0.015)$. The median targeted kidney size was $10.0(9.0-10.2) \mathrm{cm}$ in low-risk group and $9.8(9.3-10.2)$ $\mathrm{cm}$ in the high-risk group. Bilateral renal artery stent insertion was performed in 2 subjects $(7.7 \%)$ in the low-risk group and in 6 subjects (28.6\%) in the high-risk group.

\section{Kidney function improvement after PTA/S}

Among the included patients, 4 subjects were lost to follow-up or death within 4 months from baseline. Among the remaining 43 subjects, 2 subjects (8.0\%) and 5 subjects $(27.8 \%)$ showed kidney function improvement after PTA/S in the low- $(\mathrm{n}=25)$ and high-risk $(\mathrm{n}=18)$ groups, respectively $(P=0.11)$. The proportion of subjects with kidney function improvement increased in those presenting with rapid decline of kidney function (3 out of $5,60 \%$; low-risk group vs. rapid decline, $P=0.022$ ) (Fig. 1A). The eGFR improved from $28(10-45) \mathrm{mL} / \mathrm{min} / 1.73$ $\mathrm{m}^{2}$ to $41(16-67) \mathrm{mL} / \mathrm{min} / 1.73 \mathrm{~m}^{2}$ at 4 months after PTA/ $\mathrm{S}$ in subjects with rapid decline of kidney function, although the difference was not statistically significant $(P=$ 0.084) (Fig. 2). However, the eGFR did not improve after $\mathrm{PTA} / \mathrm{S}$ in the low-risk group $\left(52[42-62] \mathrm{mL} / \mathrm{min} / 1.73 \mathrm{~m}^{2}\right.$ at baseline vs. $51[42-60] \mathrm{mL} / \mathrm{min} / 1.73 \mathrm{~m}^{2}$ at 4 months, $P$ $=0.541)$. The time-dependent changes of eGFR between the low-risk group and the rapid decline of kidney func-

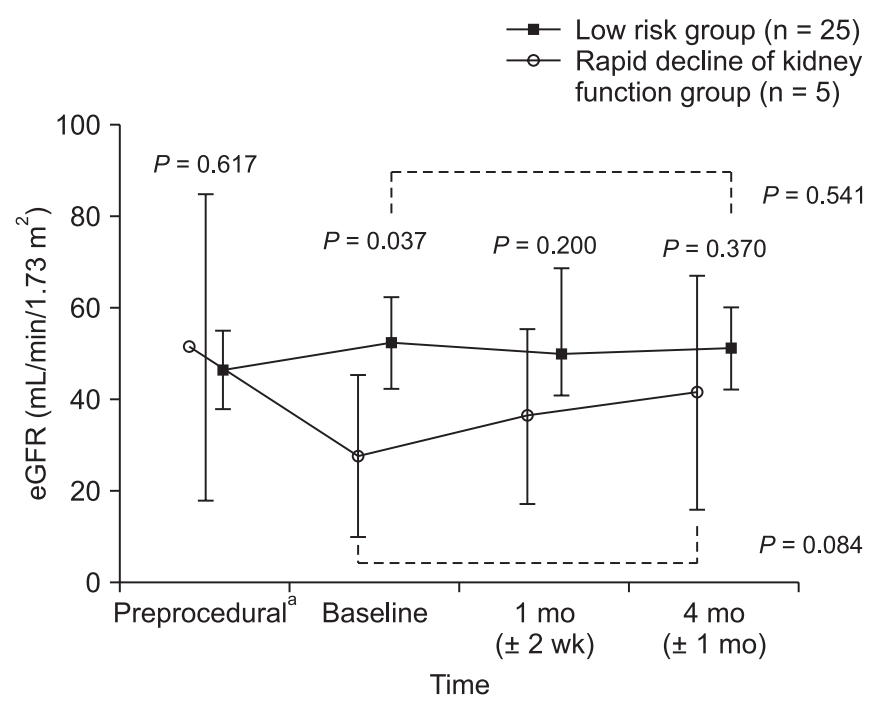

Figure 2. Change in estimated glomerular filtration rate (eGFR) after renal revascularization.

${ }^{a}$ The preprocedural eGFR is the result of the last examination within 3 months before the percutaneous transluminal angioplasty with stent insertion. tion group were significant when adjusted for age, sex, baseline eGFR, SBP, and proteinuria $(P=0.001)$.

\section{Hypertension improvement after PTA/S}

Nine (36.0\%) and 14 (77.8\%) subjects showed a reduction in BP after PTA/S in the low- $(\mathrm{n}=25)$ and high-risk ( $\mathrm{n}=18$ ) group, respectively $(P=0.007)$. The proportion of subjects with hypertension improvement further increased in the refractory hypertension group (12 out of 15, 80.0\%; low-risk group vs. refractory hypertension group, $P=0.007$ ) (Fig. 1B). In subjects who presented with refractory hypertension, SBP decreased from 157 (150-164) mmHg at baseline to $140(131-148) \mathrm{mmHg}$ at 4 months after PTA/S $(P=0.005)$. In the low-risk group, there was a slight decrease in SBP (133 [129-138] mmHg at baseline compared to 131 [124-138] $\mathrm{mmHg}$ at 4 months after PTA/S, $P=0.561$ ). There was also a decrease in BPMS in the refractory hypertension group from 3.6 $(2.1-5.0)$ at baseline to $2.8(1.6-3.9)$ at 4 months $(P=$ 0.050). Conversely, the BPMS changed marginally from $2.0(1.5-2.6)$ at baseline to $1.7(1.0-2.5)$ at 4 months in the low-risk group $(P=0.390)$ (Fig. 3$)$. There was a significant difference in the time-dependent change of SBP $(P=0.001)$ between the low-risk group and the refractory hypertension group when adjusted for age, sex, baseline eGFR, SBP, and proteinuria. However, there was no difference in the time-dependent change of DBP $(P=0.061)$ or BPMS $(P=0.627)$ between the low-risk group and the refractory hypertension group when adjusted for age, sex, baseline eGFR, SBP, and proteinuria.

\section{Outcomes of the pulmonary edema group}

Two subjects presented with pulmonary edema. One subject was stable without recurrent pulmonary edema until one month after PTA/S when she was lost to followup, while pulmonary edema did not recur in the other subject until 4 months after PTA/S.

\section{Clinical characteristics of patients with response after PTA/S}

Overall, 25 subjects (58.1\%) achieved either kidney function improvement or hypertension improvement and were therefore classified as responders. Table 2 


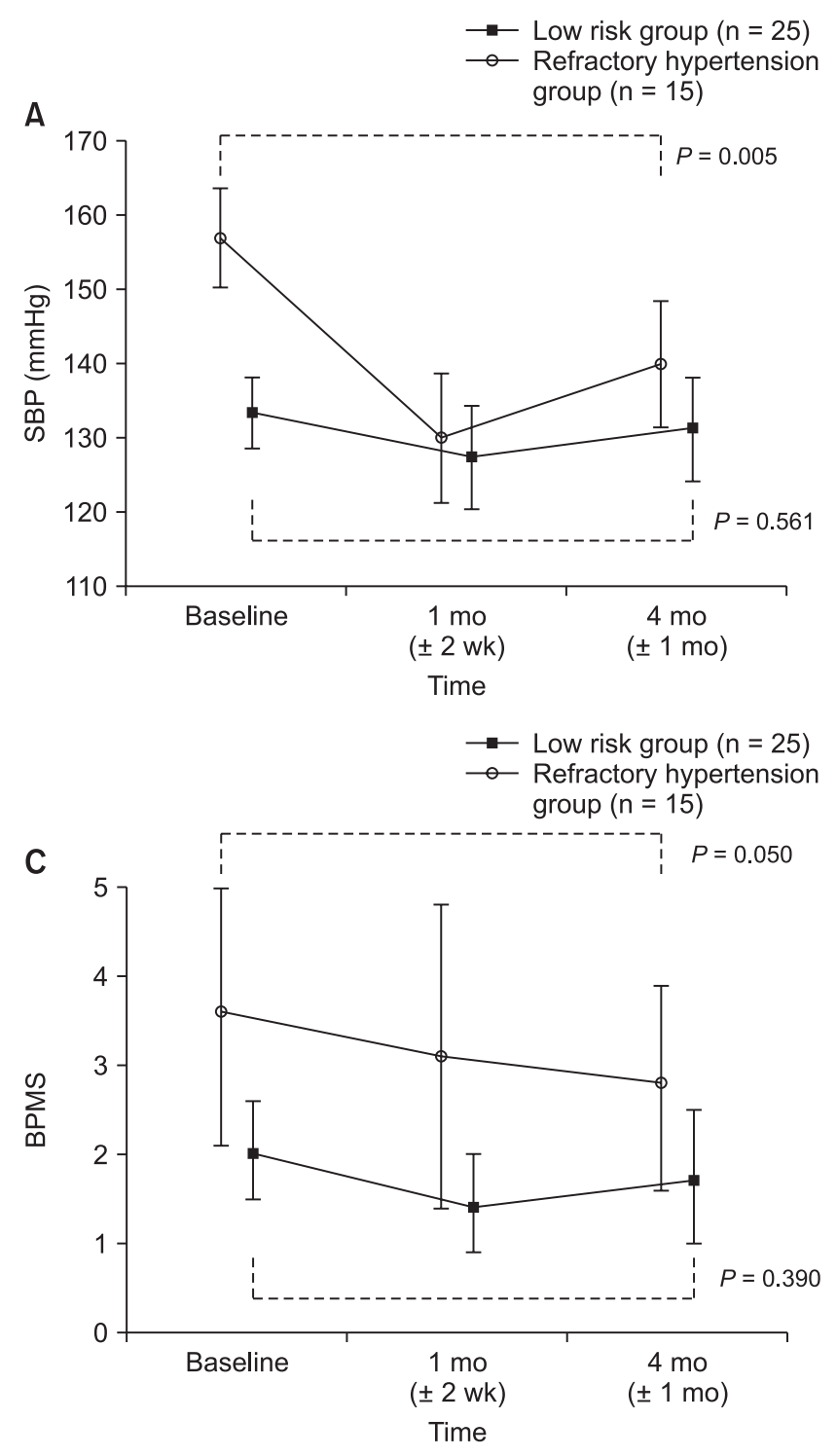

shows the characteristics of responders after PTA/S. The demographic findings of age, sex, history of smoking, BMI, and history of medication showed no difference between responders and non-responders. The baseline SBP was higher in responders than in non-responders $(P$ $=0.047)$. The proportion of subjects who presented with refractory hypertension was higher in responders than in non-responders $(P=0.006)$. All patients with rapidly declining kidney function before PTA/S showed a response after the procedure, although the difference was not significant $(P=0.064)$. The baseline eGFR showed no difference between responders and non-responders (39 [24-58] vs. 44 [30-58], $P=0.961)$. Among patients with baseline eGFR $<30 \mathrm{~mL} / \mathrm{min} / 1.73 \mathrm{~m}^{2}(\mathrm{n}=11), 7$ subjects $(63.6 \%)$ met the criteria for responders. There was no dif-

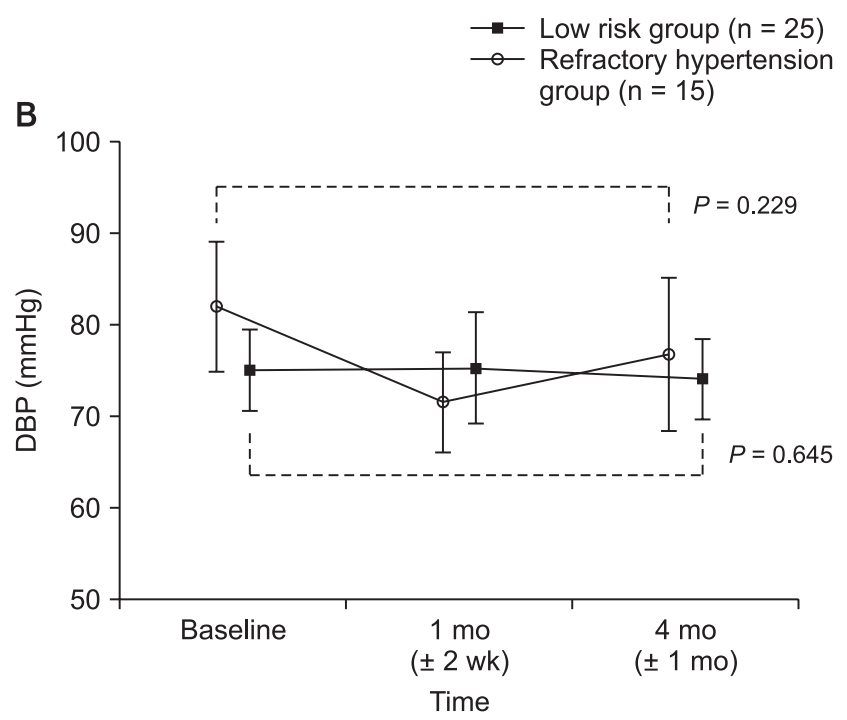

Figure 3. Changes in systolic blood pressure (SBP) (A), diastolic blood pressure (DBP) (B), and blood pressure medical score (BPMS) (C) after revascularization.

ference in the presence of baseline proteinuria between responders and non-responders $(P=0.679)$. Among patients with the targeted kidney size of 7.5 to $8.5 \mathrm{~cm}$ ( $\mathrm{n}=$ 5), 3 subjects $(60.0 \%)$ met the criteria for responders. The level of targeted arterial stenosis was associated with the response after PTA/S $(P=0.040)$. None of the patients with targeted arterial stenosis less than $75 \%(n=4)$ met the response criteria after PTA/S. When patients with less than $75 \%$ stenosis were excluded, the responder group showed a larger proportion of high-risk patients than the non-responder group (responders, 15/25 [60.0\%]; nonresponders, $3 / 14$ [21\%]; $P=0.020$ ), and the proportion of subjects who presented with refractory hypertension (responders, 13/25 [52\%]; non-responders, 2/14 [14\%]; $P=0.020$ ) or rapid decline of kidney function (respond- 
Table 2. Clinical characteristics by response to percutaneous transluminal angioplasty with stent insertion

\begin{tabular}{|c|c|c|c|}
\hline Variable & Responder $(n=25)$ & Non-responder $(n=18)$ & $P$ value \\
\hline Age (yr) & $66(59-75)$ & $69(58-75)$ & 0.803 \\
\hline Sex, male & $20(80.0)$ & $12(66.7)$ & 0.480 \\
\hline Smoking & $12(48.0)$ & $6(33.3)$ & 0.336 \\
\hline Body mass index $\left(\mathrm{kg} / \mathrm{m}^{2}\right)$ & $24.1(21.8-25.5)$ & $23.2(21.9-26.3)$ & 0.674 \\
\hline \multicolumn{4}{|l|}{ Concurrent medication } \\
\hline ACEi/ARB & $11(44.0)$ & $5(27.8)$ & 0.278 \\
\hline Diuretics & $12(48.0)$ & $11(61.1)$ & 0.553 \\
\hline CCB & $16(64.0)$ & $13(72.2)$ & 0.570 \\
\hline Beta blocker & $17(68.0)$ & $12(66.7)$ & 0.927 \\
\hline Alpha blocker & $2(8.0)$ & $1(5.6)$ & 1.000 \\
\hline Statin & $16(64.0)$ & $15(83.3)$ & 0.163 \\
\hline Aspirin & $19(76.0)$ & $11(61.1)$ & 0.294 \\
\hline Clopidogrel & $12(48.0)$ & $6(33.3)$ & 0.336 \\
\hline Other antiplatelet & $1(4.0)$ & $1(5.6)$ & 1.000 \\
\hline Warfarin & $5(20.0)$ & $5(27.8)$ & 0.717 \\
\hline SBP (mmHg) & $150(132-156)$ & $138(125-145)$ & 0.047 \\
\hline $\mathrm{DBP}(\mathrm{mmHg})$ & $80(69-89)$ & $74(68-80)$ & 0.054 \\
\hline BPMS & $2.3(1.4-4.0)$ & $1.8(1.0-3.0)$ & 0.190 \\
\hline Baseline eGFR (mL/min/1.73 $\left.\mathrm{m}^{2}\right)$ & $39(24-58)$ & $44(30-58)$ & 0.961 \\
\hline$<30 \mathrm{~mL} / \min / 1.73 \mathrm{~m}^{2}$ & $7(28.0)$ & $4(22.2)$ & 0.736 \\
\hline$\triangle \mathrm{eGFR}^{\mathrm{a}}$ & $-3.9(-10.7$ to -0.7$)$ & $-1.3(-4.2$ to -1.7$)$ & 0.050 \\
\hline uPCR (mg/mgCr) & $0.20(0.08-0.42)$ & $0.31(0.06-0.95)$ & 0.117 \\
\hline Proteinuria $^{\mathrm{b}}(\mathrm{g} / \mathrm{gCr})$ & & & 0.679 \\
\hline$<1$ & $20(87.0)$ & $14(77.8)$ & \\
\hline$\geq 1$ & $3(13.0)$ & $4(22.2)$ & \\
\hline Contralateral kidney size $^{c}(\mathrm{~cm})$ & $10.0(9.1-10.0)$ & $9.4(8.9-10.0)$ & 0.379 \\
\hline $7.5-8.5$ & $3(17.6)$ & $2(15.4)$ & 1.000 \\
\hline Targeted kidney size (cm) & $9.9(9.3-10.1)$ & $10.0(8.9-10.2)$ & 0.959 \\
\hline $7.5-8.5$ & $2(8.0)$ & $2(11)$ & 1.000 \\
\hline Targeted arterial stenosis $^{d}$ & $89(80-95)$ & 85 (74-95) & 0.183 \\
\hline$>95 \%$ & $3(12.5)$ & $2(11.1)$ & \\
\hline $75-95 \%$ & $21(87.5)$ & $12(66.7)$ & 0.040 \\
\hline$<75 \%$ & $0(0)$ & $4(22.2)$ & \\
\hline Bilateral stent insertion & $5(20.0)$ & $3(16.7)$ & 1.000 \\
\hline Low-risk/high-risk & $10(40.0) / 15(60.0)$ & $15(83.3) / 3(16.7)$ & 0.004 \\
\hline Refractory hypertension & $13(52.0)$ & $2(11.1)$ & 0.006 \\
\hline Rapid decline of kidney function & $5(20.0)$ & $0(0)$ & 0.064 \\
\hline
\end{tabular}

Data are presented as median (interquartile range) or number (\%).

ACEi, angiotensin-converting enzyme inhibitor; ARB, angiotensin receptor blocker; BPMS, blood pressure medical score; CCB, calcium channel blocker; DBP, diastolic blood pressure; SBP, systolic blood pressure; eGFR, estimated glomerular filtration rate; UPCR, urine protein-to-creatinine ratio.

${ }^{a}$ Median change in kidney function between baseline eGFR and pre-procedural eGFR. The pre-procedural eGFR is the result of the last examination within six months prior to the PTA/S.

${ }^{b}$ Data regarding baseline UPCR were not routinely collected. Total number of responders was 23 due to missing data for two subjects.

'Data regarding contralateral kidney size were not routinely measured. Total numbers of responders and non-responders were 17 and 13 , respectively, due to missing data for 13 subjects.

${ }^{d}$ Data regarding targeted arterial stenosis were not routinely collected. Total number of responders was 24 due to missing data for one subject.

$P$ values were calculated using either the Mann-Whitney $U$ test or Fisher's exact test. 
ers, 5/25 [20\%]; non-responders, $0 / 14$ [0\%]; $P=0.073$ ) was higher in responders than in non-responders.

\section{Procedure-related complications}

PTA/S-related complications were reported in 5 of the 47 patients (10.6\%): cholesterol embolism in one patient, catheter puncture site haematoma in a second patient, and renal artery dissections in the remaining. Among the 47 patients who underwent PTA/S, post-PTA acute kidney injury occurred in 8 subjects (17.0\%).

\section{Discussion}

The present study demonstrated that patients with ARAS presenting with high-risk clinical manifestations might benefit from revascularization, which is consistent with previous studies $[9,22]$. We found that subjects with rapid deterioration of kidney function showed kidney function improvement, and those with refractory hypertension achieved better control of $\mathrm{BP}$, with a decrease in the number of antihypertensive medications after $\mathrm{PTA} / \mathrm{S}$, compared to the low-risk group. In addition, this study suggested that, even if baseline eGFR is $<30 \mathrm{~mL} /$ $\min / 1.73 \mathrm{~m}^{2}, \mathrm{PTA} / \mathrm{S}$ may be considered as a treatment option in patients with clinically significant ARAS.

Optimal medical treatment without revascularization has been the 'gold standard' for low-risk and stable ARAS, according to a previously reported RCT and metaanalysis [10]. Recently, the two largest RCTs evaluating percutaneous renal artery intervention for RAS, the CORAL trial and the Angioplasty and Stenting for Renal Artery Lesions (ASTRAL) trial, demonstrated that revascularization did not offer significant benefits over medical therapy in patients with ARAS and stable chronic kidney disease $[3,4]$. These trials share the limitation of excluding subsets of patients with high-risk clinical presentations, such as episodic pulmonary edema, rapid decline of kidney function, and hypertension [23].

In the ASTRAL trial [3], the primary outcome measure was change in renal function over time. During a median follow-up period of 34 months, the rate of progression of renal impairment differed, being lower in the revascularization group at $0.06 \times 10^{-3} \mathrm{~L} / \mu \mathrm{mol}$ per year $(95 \%$ confidence interval, -0.002 to $0.13 ; P=0.06$ ). There were no between-group differences in the following secondary outcomes: SBP/DBP, rates of renal events, major cardiovascular events, and death. However, the majority of the patients in the ASTRAL trial had only mild-to-moderate RAS, and about $40 \%$ of subjects had a stenosis $<50 \%$ and were least likely to benefit from revascularization. Twenty-five percent of subjects had normal renal function and a further $15 \%$ almost-normal renal function at baseline. The aim of the CORAL trial [4] was to evaluate "hard" clinical endpoints using cardiovascular or renal events. Over a median follow-up period of 43 months, there were no significant differences between the treatment groups in primary outcomes and overall survival. The original study protocol included patients with $60 \%$ to $100 \%$ RAS on angiography, with an SBP above $155 \mathrm{mmHg}$, and taking two or more anti-hypertensive medications. However, there have been changes in the original inclusion criteria due to slow enrollment. As a result, $25 \%$ to $30 \%$ of patients have shown well-controlled BP, $55 \%$ of subjects had $<70 \%$ stenosis, and approximately $50 \%$ of the cohort presented normal kidney function.

There have been several studies reporting the benefits of revascularization in high-risk patients with ARAS $[8,9,22,24]$. Patients presenting with a combination of rapidly declining kidney function and refractory hypertension may benefit from revascularization [22]. Vassallo et al. [9] have recently demonstrated that high-risk patients, especially those presenting with rapidly deteriorating renal function and concurrent bilateral $\geq 70 \%$ RAS and low levels of proteinuria ( $<1 \mathrm{~g} /$ day $)$ at the time of diagnosis, benefited from revascularization. In their study, high-risk patients were defined as those presenting with $\geq 70 \%$ unilateral or bilateral angiographic RAS with at least one high-risk clinical manifestation such as flash pulmonary edema, stage 2 hypertension, or rapidly deteriorating renal function. The study used stricter inclusion criteria for uncontrolled or severe hypertension (SBP $\geq 160 \mathrm{mmHg}$ and/or DBP $\geq 100 \mathrm{mmHg}$ ) than the present study. In addition, the definition of rapidly declining kidney function differed from that of the present study, as the eGFR slope was defined as less than -3.0 $\mathrm{mL} / \mathrm{min} / 1.73 \mathrm{~m}^{2} /$ year. In our study, $58 \%$ of subjects with baseline uPCR $<1 \mathrm{mg} / \mathrm{mg} \mathrm{Cr}$ showed an improvement in kidney function or better control of BP. None of the patients with baseline uPCR $>1 \mathrm{~g} / \mathrm{g}$ Cr showed any increase in kidney function after PTA/S (data not shown), but this was not statistically significant, which was likely due to 
the limited number of patients. Proteinuria may imply the presence of comorbid renal parenchymal disease $[25,26]$. In this circumstance, the revascularization of RAS may have little impact on the outcome of kidney disease. In addition, none of the patients with a targeted arterial stenosis $<75 \%$ showed any benefit on revascularization in the present study. These results are consistent with the finding that a RAS greater than $70 \%-80 \%$ is necessary to activate intra-renal RAS [23], although identification of haemodynamic significance by anatomical stenotic severity is limited [11,27-29].

There are several limitations to be mentioned. First, as this was a retrospective study, patients were not randomized to revascularization; hence, selection bias and potential hidden confounders may affect the results. However, considering that recruitment to renal stenting trials is very challenging (e.g., the recruitment phase in the ASTRAL study lasted seven years and approximately two patients per year were enrolled) [3], our findings based on retrospective data are worthy of attention. Second, this study was a small single-arm registry study without comparison with an optimal medical therapy arm. Thus, the generalizability of our findings remains to be determined. Third, because of the relatively short follow-up period, we did not examine the effects of revascularization on the long-term effects on the kidney or the patient. Instead, immediate changes in BP and kidney function were evaluated in detail.

The present study investigated the clinical efficacy of revascularization in patients with ARAS and suggested beneficial effects of revascularization on hypertension and kidney function in patients who presented with highrisk clinical features. Patients with stenosis $<75 \%$ showed poorer responses to PTA/S, while an advanced chronic kidney disease stage was not a predictor of poor response to PTA/S. Two recent RCTs of PTA for ARAS failed to demonstrate any advantages on renal protection or prevention of cardiovascular events compared with conventional medical therapy. This study implied that the optimal application of PTA/S should be based on individual assessment of the clinical significance of RAS.

\section{Conflicts of interest}

All authors have no conflicts of interest to declare.

\section{Authors' contributions}

Jung Eun Lee was involved in study design, data collection, analysis, interpretation of results and review of the manuscript. Suhyun Kim and Mi Jeoung Kim were involved in data collection, analysis, interpretation of results and writing of the manuscript. Jeunseok Jeon and Hye Ryoun Jang contributed to conception, analysis and interpretation of data. Kwang Bo Park and Wooseong Huh and Young Soo Do were involved in data collection and reviewed the manuscript and provided comments. YoonGoo Kim and Dae Joong Kim and Ha Young Oh contributed to analysis and interpretation of data and revision of the article for important scientific content. All the authors read and approved the final version of the manuscript.

\section{References}

[1] Derkx FH, Schalekamp MA. Renal artery stenosis and hypertension. Lancet 1994;344:237-239.

[2] Ram CV. Renovascular hypertension. Curr Opin Nephrol Hypertens 1997;6:575-579.

[3] ASTRAL Investigators, Wheatley K, Ives N, et al. Revascularization versus medical therapy for renal-artery stenosis. N Engl J Med 2009;361:1953-1962.

[4] Cooper CJ, Murphy TP, Cutlip DE, et al; CORAL Investigators. Stenting and medical therapy for atherosclerotic renal-artery stenosis. N Engl J Med 2014;370:13-22.

[5] Kumbhani DJ, Bavry AA, Harvey JE, et al. Clinical outcomes after percutaneous revascularization versus medical management in patients with significant renal artery stenosis: a meta-analysis of randomized controlled trials. Am Heart $J$ 2011;161:622-630.e1.

[6] Jenks S, Yeoh SE, Conway BR. Balloon angioplasty, with and without stenting, versus medical therapy for hypertensive patients with renal artery stenosis. Cochrane Database Syst Rev 2014;(12):CD002944.

[7] Hirsch AT, Haskal ZJ, Hertzer NR, et al; American Association for Vascular Surgery/Society for Vascular Surgery; Society for Cardiovascular Angiography and Interventions; Society for Vascular Medicine and Biology; Society of Interventional Radiology; ACC/AHA Task Force on Practice Guidelines. ACC/AHA Guidelines for the Management of Patients with Peripheral Arterial Disease (lower extremity, renal, mesenteric, and abdominal aortic): a collaborative report from the American Associations for Vascular Sur- 
gery/Society for Vascular Surgery, Society for Cardiovascular Angiography and Interventions, Society for Vascular Medicine and Biology, Society of Interventional Radiology, and the ACC/AHA Task Force on Practice Guidelines (writing committee to develop guidelines for the management of patients with peripheral arterial disease)--summary of recommendations. J Vasc Interv Radiol 2006;17:1383-1397.

[8] Ma Z, Liu L, Zhang B, Chen W, Yang J, Li H. Renal artery stent in solitary functioning kidneys: $77 \%$ of benefit: a systematic review with meta-analysis. Medicine (Baltimore) 2016;95:e4780.

[9] Vassallo D, Ritchie J, Green D, Chrysochou C, Kalra PA. The effect of revascularization in patients with anatomically significant atherosclerotic renovascular disease presenting with high-risk clinical features. Nephrol Dial Transplant 2018;33:497-506.

[10] Karanikola E, Karaolanis G, Galyfos G, Barbaressos E, Palla V, Filis K. Endovascular management of atherosclerotic renal artery stenosis: post-cardiovascular outcomes in renal atherosclerotic lesions era winner or false alarm? Vasc Specialist Int 2017;33:1-15.

[11] Manolis AS, Manolis AA, Melita H. Current status of renal artery angioplasty and stenting for resistant hypertension: a case series and review of the literature. Curr Hypertens Rev 2017;13:93-103.

[12] Inker LA, Schmid CH, Tighiouart H, et al.; CKD-EPI Investigators. Estimating glomerular filtration rate from serum creatinine and cystatin C. N Engl J Med 2012;367:20-29.

[13] Levy PD, Willock RJ, Burla M, et al. Total antihypertensive therapeutic intensity score and its relationship to blood pressure reduction. J Am Soc Hypertens 2016;10:906-916.

[14] Comparison of angiotensin converting enzyme (ACE) inhibitors [Internet]. Vancouver: Vancouver Acute, c2008 [cited 2018 Jul 1]. Available from: http://www.vhpharmsci. com/vhformulary/Tools/ACEI\%20Comparison.pdf.

[15] Angiotensin II receptor blockers [Internet]. Detroit, MI: GlobalRPh Inc., 2017 [cited 2018 Jul 1]. Available from: http://globalrph.com/drugs/angiotensin-ii-receptorblockers.

[16] Drug comparisons - beta blockers equivalent doses [Internet]. Detroit, MI: GlobalRPh Inc., 2017 [cited 2018 Jul 1]. Available from: http://globalrph.com/medcalcs/betablockers/.

[17] Dihydropyridine calcium channel blocker interchange [Internet]. Vancouver: Vancouver Acute, c2008 [cited 2018 Jul 1]. Available from: http://www.vhpharmsci.com/vhformu-
lary/Tools/CCB\%20Interchange.pdf.

[18] Rossetti G, Pizzocri S, Brasca F, et al. Antihypertensive effect of barnidipine $10 \mathrm{mg}$ or amlodipine 5 to $10 \mathrm{mg}$ once daily in treatment-naive patients with essential hypertension: A 24-week, randomized, open-label, pilot study. Curr Ther Res Clin Exp 2008;69:192-206.

[19] UK Medicines Information. What are appropriate doses to use when switching hypertensive patients to amlodipine from lercanidipine or lacidipine? [Internet]. Redhill (UK): Surrey and Sussex Healthcare NHS Trust, 2012 [cited 2018 Jul 1]. Available from: https://www.surreyandsussex.nhs. uk/wp-content/uploads/2013/04/UKMi-Switching-dosesfor-amlodipine-CCBs1.pdf.

[20] Harada K, Ohmori M, Fujimura A. Comparison of the antagonistic activity of tamsulosin and doxazosin at vascular alpha 1-adrenoceptors in humans. Naunyn Schmiedebergs Arch Pharmacol 1996;354:557-561.

[21] Mancia G, Fagard R, Narkiewicz K, et al. 2013 ESH/ESC guidelines for the management of arterial hypertension: the Task Force for the Management of Arterial Hypertension of the European Society of Hypertension (ESH) and of the European Society of Cardiology (ESC). Eur Heart $J$ 2013;34:2159-2219.

[22] Ritchie J, Green D, Chrysochou C, Chalmers N, Foley RN, Kalra PA. High-risk clinical presentations in atherosclerotic renovascular disease: prognosis and response to renal artery revascularization. Am J Kidney Dis 2014;63:186-197.

[23] Mohan IV, Bourke V. The management of renal artery stenosis: an alternative interpretation of ASTRAL and CORAL. Eur J Vasc Endovasc Surg 2015;49:465-473.

[24] Fujihara M, Yokoi Y, Abe T, et al; J-RAS Study Investigators. Clinical outcome of renal artery stenting for hypertension and chronic kidney disease up to 12 months in the J-RAS Study - prospective, single-arm, multicenter clinical study. Circ J 2015;79:351-359.

[25] Hemmelgarn BR, Manns BJ, Lloyd A, et al.; Alberta Kidney Disease Network. Relation between kidney function, proteinuria, and adverse outcomes. JAMA 2010;303:423-429.

[26] Gerstein HC, Mann JF, Yi Q, et al.; HOPE Study Investigators. Albuminuria and risk of cardiovascular events, death, and heart failure in diabetic and nondiabetic individuals. JAMA 2001;286:421-426.

[27] Leesar MA, Varma J, Shapira A, et al. Prediction of hypertension improvement after stenting of renal artery stenosis: comparative accuracy of translesional pressure gradients, intravascular ultrasound, and angiography. J Am Coll Car- 
diol 2009;53:2363-2371.

[28] Mitchell JA, Subramanian R, White CJ, et al. Predicting blood pressure improvement in hypertensive patients after renal artery stent placement: renal fractional flow reserve. Catheter Cardiovasc Interv 2007;69:685-689.
[29] Kądziela J, Januszewicz A, Prejbisz A, et al. Prognostic value of renal fractional flow reserve in blood pressure response after renal artery stenting (PREFER study). Cardiol $J$ 2013;20:418-422. 